\title{
Reversal mechanism of submicron patterned CoNi/Pt multilayers
}

\author{
M. A. M. Haast, J. R. Schuurhuis, L. Abelmann, J. C. Lodder and Th. J. Popma \\ MESA Research Institute, University of Twente, P.O.Box 217, 7500 AE Enschede, The Netherlands
}

\begin{abstract}
With laser interference lithography $\mathrm{Co}_{50} \mathrm{Ni}_{50} / \mathrm{Pt}$ multilayers with perpendicular magnetic anisotropy have been patterned into regular matrices of submicron sized dots. Their magnetic properties have been studied with Vibrating Sample Magnetometry. Compared to continuous multilayers $\left(H_{c}=15\right.$ $\mathrm{kA} / \mathrm{m})$ the coercivity for the patterned multilayers is much larger $\left(H_{c}=115-270 \mathrm{kA} / \mathrm{m}\right)$. Though the hysteresis curves of $180 \mathrm{~nm}$ dots and $60 \mathrm{~nm}$ dots have identical shapes, virgin curves seem to indicate that the $180 \mathrm{~nm}$ dots are multidomain while the $60 \mathrm{~nm}$ dots are single domain. The latter has been confirmed with Magnetic Force Microscopy observations.
\end{abstract}

Index Terms-Discrete magnetic recording, Laser interference lithography, Magnetic multilayers, Reversal behaviour.

\section{INTRODUCTION}

For future ultra high density magnetic recording the medium has to be patterned into single domain dots where every dot represents one bit [1]. Therefore patterned magnetic materials are attracting a lot of interest [2], [3]. A requirement for the patterning technique to be used in mass production is that large areas can be patterned into submicron dots. Laser interference lithography meets this requirement [4], [5] and is applied in our present work to study the magnetic properties of submicron dots. Requirements for the magnetic material in a discrete recording medium are that it has an high uniaxial magnetic anisotropy and a strong intergranular coupling within one dot (for polycrystalline materials). $\mathrm{Co}_{50} \mathrm{Ni}_{50} / \mathrm{Pt}$ multilayers can exhibit both properties. For thin $\mathrm{CO}_{50} \mathrm{Ni}_{50}$ layers $(<10 \AA)$ these layers can have a perpendicular magnetic anisotropy and a low coercivity indicating strong intergranular coupling. It is obvious that the reversal behaviour of submicron patterned $\mathrm{Co}_{50} \mathrm{Ni}_{50} / \mathrm{Pt}$ multilayers is a very interesting subject of study. In this paper the patterning process is described in detail and a first comparison is made between the reversal of continuous and patterned $\mathrm{CO}_{50} \mathrm{Ni}_{50} / \mathrm{Pt}$ multilayers with dot sizes of 180 and $60 \mathrm{~nm}$. Moreover, the domain state (single- or multidomain) of the dots is investigated.

\section{Patterning PROCESS}

The $\mathrm{Co}_{50} \mathrm{Ni}_{50} / \mathrm{Pt}$ multilayers are deposited by sputtering on thermally oxidized 3" Si wafers. The sputtering system ( $p_{\text {basse }}$ $=5 \cdot 10^{-8}$ mbar) has a rotating substrate table and two magnetron guns with 2 " targets, equiped with a shutter system. The sputtering gas is Argon and the deposition pressure is 12 $\mu$ bar. The total multilayer structure consists of 26 bilayers of $6 \AA \mathrm{Pt}$ and $6 \AA \mathrm{CO}_{50} \mathrm{Ni}_{50}$. For protection against oxidation, on top an extra $20 \AA \mathrm{Pt}$ layer is deposited. The total thickness of

Manuscript received October $14,1997$.

M. A. M. Haast $+31-53-4892710$, fax $+31-53-4893343$, m.a.m.hanst@eltn.utwente.nl, http://www.el.utwente.nl/tdm/istg.

This work was supported by the Dutch Foundation of Technology (STW). the multilayer is then $33 \mathrm{~nm}$. The total effective perpendicular magnetic anisotropy of this multilayer, which was measured with a torque magnetometer, equals $160 \mathrm{~kJ} / \mathrm{m}^{3}$

Next step in the process is the spinning of a $100 \mathrm{~nm}$ thick layer of positive resist on the multilayer and its exposure by an interference pattern of two glancing incident laser beams (Ar $351 \mathrm{~nm}$ ). This interference pattern is formed by a laser beam directly incident on the substrate and one reflected on a mirror which is mounted perpendicularly to the substrate. After one exposure the substrate is rotated over $90^{\circ}$ in its plane and a second exposure is carried out. Then, after development, a regular pattern of dots of resist is formed. The period of the dot pattern can be changed between 200 and 600 $\mathrm{nm}$ by varying the angle of the substrate with the laser beam. The final size of the dots is determined by the exposure dose.

Finally the resist pattern is transfered to the multilayer with ion beam etching. Argon is used as etching gas with an ion energy of $500 \mathrm{eV}$ and beam current of $12.5 \mathrm{~mA}$. For the $\mathrm{Co}_{50} \mathrm{Ni}_{50} / \mathrm{Pt}$ multilayers typical etch rates are $10 \mathrm{~nm} / \mathrm{min}$. An in-situ four point resistivity measurement is applied for end point detection.

Fig. 1 and 2 show two examples of etched dot structures. The first SEM image shows a cross section of $300 \mathrm{~nm}$ diameter dots with $600 \mathrm{~nm}$ period. The different layers present in the sample can be distinguished. At the bottom there is the silicon substrate with the siliconoxide layer. The multilayer is seen as the bright line across the dot and the top of the dots consists of a residual resist layer. The actual magnetic dots have a disk shape. The second SEM image shows an oblique view of dots with $200 \mathrm{~nm}$ period. In this case the dot height is smaller than the thickness of the deposited multilayer, which means that the multilayer has partially been etched. The magnetic dots have a pyramid shape with an average diameter of $70 \mathrm{~nm}$. Apparently for small periods, which corresponds to a small angle $\left(30^{\circ}\right)$ between the plane of the substrate and laser beam in the laser interference exposure, the disk shape of the dots can not be maintained.

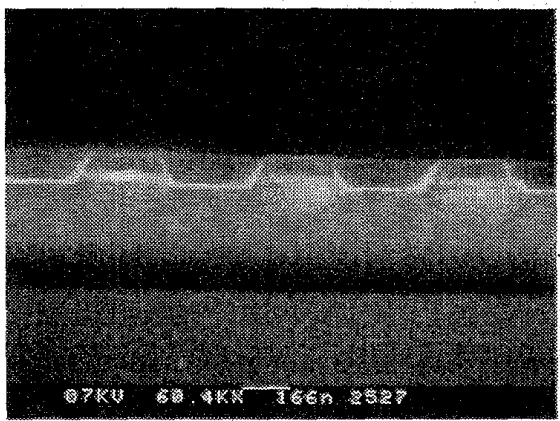

Fig. 1. SEM cross section of a patterned $\mathrm{Co}_{50} \mathrm{Ni}_{50} / \mathrm{Pt}$ multilayer with a dot period of $600 \mathrm{~mm}$. The magnetic layer can be seen as the bright line across the dot. 


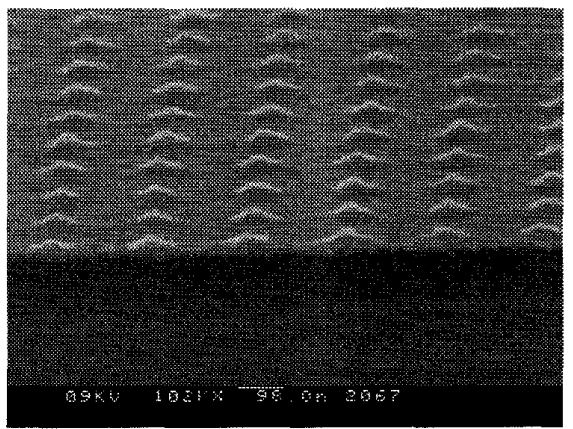

Fig. 2. Oblique SEM image of a patterned $\mathrm{CO}_{50} \mathrm{Ni}_{50} / \mathrm{Pt}$ multilayer with a dot period of $200 \mathrm{~nm}$. The magnetic layer has partially been etched and the dots have a pyramid shape.

For comparison also micron sized dots have been prepared with standard optical lithography.

\section{MAGNETIC CHARACTERIZATION}

The magnetic reversal has been studied with an Oxford Vibrating Sample Magnetometer (VSM). Perpendicular hysteresis curves have been measured with a maximum applied field of $1000 \mathrm{kA} / \mathrm{m}$. Virgin curves have been measured after inplane demagnetization. The demagnetization started from saturation and took place by sweeping the field several times between positive and negative direction while reducing its amplitude. Magnetic Force Microscopy (MFM) observations have been carried out with a home built MFM [6].

\section{MAGNETIC PROPERTIES}

\section{A. $\mathrm{Co}_{50} \mathrm{Ni}_{50}$ Pt multilayers}

Before discussing magnetic properties of submicron patterned $\mathrm{Co}_{50} \mathrm{Ni}_{50} / \mathrm{Pt}$ multilayers it is important to understand the magnetic properties of the continuous multilayers. Fig. 3 shows the hysteresis curve of a $\left\{\mathrm{Co}_{50} \mathrm{Ni}_{50}(6 \AA) / \mathrm{Pt}(6 \AA)\right\} \times 26$ multilayer. From saturation the magnetic moment drastically drops at around zero applied field and then slowly increases to saturation in the opposite direction. As discussed by other authors this reversal behaviour can be explained by two processes [7], [8]. First at the nucleation point stripe domains are formed and the sample comes in a demagnetised state. Note that this means that the energy barriers for domain wall displacement are very small. Then when further increasing the applied field, the stripe domains are narrowed and completely vanish at saturation. Moreover, the origin of the initial nucleation is the presence of small, vestigial domains which become unstable at some certain applied field. When the density of these vestigial domains is small compared to the dot density in the patterned $\mathrm{Co}_{50} \mathrm{Ni}_{50} / \mathrm{Pt}$ multilayers, the stripe domain state can not be formed inside all the dots and the reversal behaviour will be different irrespective of the question whether the dots are single- or multidomain.

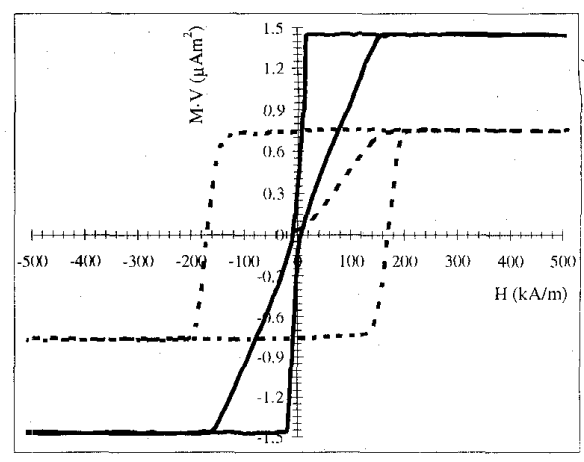

Fig. 3. VSM hysteresis curve for a $1 \times 1 \mathrm{~cm}^{2}$ continuous $\left\{\mathrm{Co}_{50} \mathrm{Ni}_{50}(6 \AA) / \mathrm{Pt}(6\right.$ $\AA$ ) ) $\times 26$ multilayer (drawn line) and VSM hysteresis and virgin curve of $1 \times 1$ $\mathrm{cm}^{2}$ sample with $2.6 \times 2.6 \mu \mathrm{m}^{2}\left\{\mathrm{Cos}_{51} \mathrm{Ni}_{s 0}(6 \AA) / \mathrm{Pt}(6 \AA)\right\} \times 26$ multilayer dots with $4 \mu \mathrm{m}$ period (dashed line).

Indeed, even for $2.6 \times 2.6 \mu \mathrm{m}^{2}\left\{\mathrm{Co}_{50} \mathrm{Ni}_{50}(6 \AA) / \mathrm{Pt}(6 \AA)\right\} \times 26$ multilayer dots with $4 \mu \mathrm{m}$ period the hysteresis drastically changes with respect to the continuous multilayer (see Fig. 3). The dots exhibit $100 \%$ remenance and the switching occurs in a narrow field range, which resembles a typical reversal behaviour of highly oriented single domain particles. However, the virgin curve of the dots is identical to the magnetization curve of the continuous multilayer, indicating that the dots are multidomain after in-plane demagnetization and that the energy barriers for domain wall displacement are the same for the continuous multilayer and the dots. Apparently the reversal of the multilayer dots takes place by nucleation of a reverse domain immediately followed by a domain wall movement througout the whole dot.

\section{B. $\mathrm{Co}_{50} \mathrm{Ni}_{50} / \mathrm{Pt}$ multilayer dots with $570 \mathrm{~nm}$ period}

By varying the exposure dose in the laser interference lithography $570 \mathrm{~nm}$ period $\left\{\mathrm{Co}_{50} \mathrm{Ni}_{50}(6 \AA) / \mathrm{Pt}(6 \AA)\right\} \times 26 \mathrm{mul}-$ tilayer dots with dotsizes in the range $180-280 \mathrm{~nm}$ have been prepared. For all dot sizes the hysteresis curves have similar shapes, but the coercivity varies from 160 to $270 \mathrm{kA} / \mathrm{m}$. No correlation between dot sizes and coercivity is found. For a $180 \mathrm{~nm}$ diameter dot sample the hystereses and virgin curve are shown in Fig. 4. As for the 2.6x2.6 $\mu \mathrm{m}^{2}$ dots the virgin

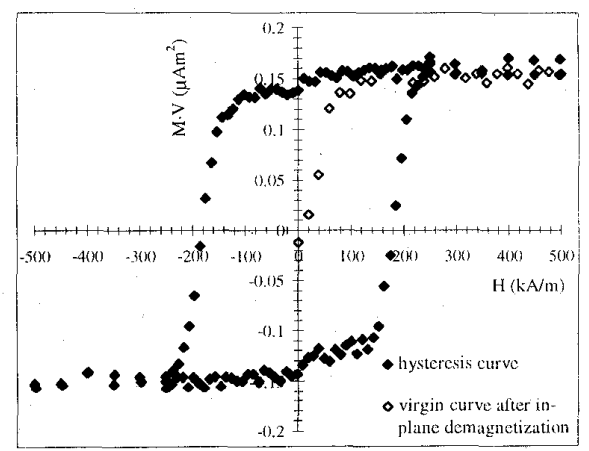

Fig. 4. VSM hysteresis and virgin curve for $\left\{\mathrm{Co}_{50} \mathrm{Ni}_{50}(6 \AA) / \mathrm{Pt}(6 \AA)\right\} \times 26$ multilayer dots with $180 \mathrm{~nm}$ diameter and $570 \mathrm{~nm}$ period. Total area of the sample is $1 \mathrm{~cm}^{2}$. 
curve is identical to the magnetization curve of the continous multilayer. The dots seem to be multidomain and the reversal seems to take place by the same mechanism as described for the $2.6 \times 2.6 \mu \mathrm{m}^{2}$ dots. The observation that there is no correlation between dot size and coercivity might indicate that the nucleation field depends on, for instance, the roughness of the dot edges or the sharpness of the dots corners, though no proof has been found yet.

\section{C. $\mathrm{Co}_{50} \mathrm{Ni}_{5}$ /Pt multilayer dots with $200 \mathrm{~nm}$ period}

Though the shape of the $200 \mathrm{~nm}$ period dots is not as perfect as that of the $570 \mathrm{~nm}$ period dots, their magnetic properties are much more interesting. Fig. 5 shows the hysteresis and virgin curve of such a $200 \mathrm{~nm}$ period dot sample. Note the extremely small signal in the VSM measurement. In fact at these small signals irreproducible background signals (e.g. signal from magnetic dust on the sample) can be significant. Probably not all background can be corrected for by simple subtraction of a background measurement. Therefore the rounding in the hysteresis curves might still have its origin in uncorrected background rather than an origin in the reversal of the dots. Taking this into account the hysteresis curve seems identical to that of the larger dot samples.

However, in this case, the virgin curve shows a different behaviour from the magnetization curve of a continous multilayer. In fact, the observed behaviour is typical for single domain particles. Additional MFM observations indicate that these dots are really single domain (see Fig. 6).

Moreover, the coercivity of the $200 \mathrm{~nm}$ period dots samples showed to be inversely proportional to the magnetic moment, from $70 \mathrm{kA} / \mathrm{m}$ for $0.1 \mu \mathrm{Am}^{2}$ to $115 \mathrm{kA} / \mathrm{m}$ for 0.06 $\mu \mathrm{Am}^{2}$. This means that with decreasing dot diameter and/or thickness the coercivity increases which is expected for single domain particles. The conclusion that these dots are single domain seems therefore justified and the reversal probably takes place by incoherent rotation.

Note that the coercivity of these single domain dots is much smaller than that of the multidomain dots, which is then remarkable. This contradiction might be related to the fact that the dots have partially been etched. Because of intermixing induced by the ion bombardment, it might well be

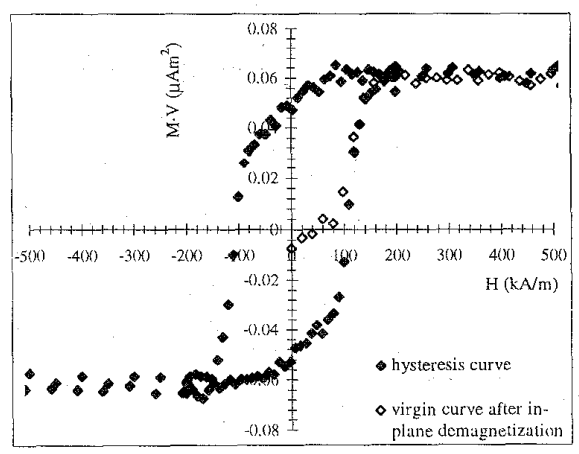

Fig. 5. VSM hysteresis and virgin curve for partially etched (see Fig. 2) $\left\{\mathrm{Co}_{50} \mathrm{Ni}_{50}(6 \AA) / \mathrm{Pt}(6 \AA)\right\} \times 26$ multilayer dots with $60 \mathrm{~nm}$ diameter and $200 \mathrm{~nm}$ period. Total area of the sample is $1 \mathrm{~cm}^{2}$.

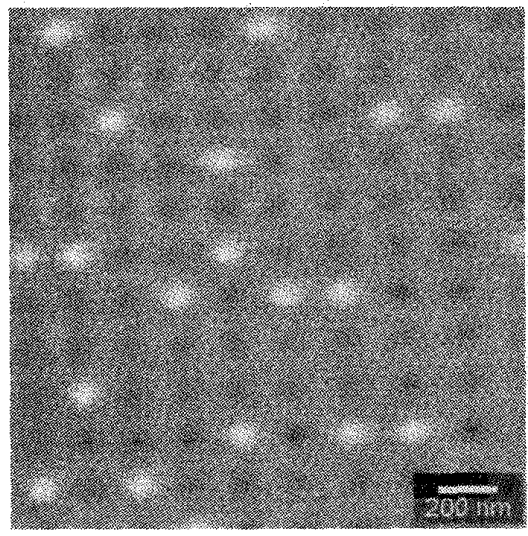

Fig. 6. Magnetic Force Microscopy image of $\mathrm{Co}_{50} \mathrm{Ni}_{50} / \mathrm{Pt}$ multilayer dots with $70 \mathrm{~nm}$ diameter and $200 \mathrm{~nm}$ period. The image was taken after demagnetization and at zero applied field.

possible that the outer region of the dots has a less perfect multilayer structure, which reduces the perpendicular anisotropy in this region and facilitates nucleation at these sites.

In order to further study the reversal of the dots, at present the activation volume (with time dependent magnetisation measurements) and the rotational hystereses loss (with torque measurements) is under investigation.

\section{CONCLUSIONS}

$\mathrm{Co}_{50} \mathrm{Ni}_{50} / \mathrm{Pt}$ multilayers with perpendicular magnetic anisotropy have been patterned into submicron dots using laser interference lithography. Down to dotsizes of $180 \mathrm{~nm}$ the dots are multidomain and reverse by nucleation followed by domain wall movement. For dotsizes smaller than $70 \mathrm{~nm}$ the dots are single domain and reverse by incoherent rotation.

\section{ACKNOWLEDGMENTS}

The authors would like to express their thanks to G. Veldhuis and H. van Wolferen for the assistance in the laser interference exposure, to I. Heskamp for general assistatice in the lithography and to S. Porthun for the MFM observations.

\section{REFERENCES}

[1] S. Y. Chou, Proceedings of the IEEE, vol. 85, pp.652-671, April 1997 . [2] J. F. Smyth, S. Schultz, D. R. Fredkin, D. P. Kern, S. A. Rishton, H. Schmid, M. Cali, T. R. Koehler, J. Appl. Phys., 69, 5262, April 1991.

[3] R. M. H. New, R. F. W. Pease and R. L. White, J. Vac. Sci. Technol. B vol. 12, pp. 3196-3201, November-December 1994.

[4] J. P. Spallas, R. D. Boyd, J. A. Britten, A. Fernandez, A. M. Hawryluk, M. D. Perry and D. R. Kania, J. Vac. Sci. Technol. B, vol. 14, pp. 20052007, May-June 1996.

[5] A. Fernandez, P. J. Bedrossian, S. L. Baker, S. P. Vernon and D. R. Kania, IEEE Trans. Mag,, vol. 32, pp. 4472-4474, September 1996.

[6] S. Porthun, M. Ruhrig, and J. C. Lodder, Forces in Scanning Probe Microscopy, NATO ASI Series: Applied Sciences, Kluwer Academic Publishers, 1995.

[7] C. Kooy and U. Enz, Philips Research Reports, vol 15, pp. 7-29, 1960.

[8] J. R. Barnes, S. J. O'Shea, M. E. Welland, J.-Y. Kim, J. E. Events and R. E. Somekh, J. Appl. Phys, vol. 76, pp. 2974-2980, September 1994. 